\title{
Trombo recurrente sobre dispositivo de cierre percutáneo de orejuela izquierda: una situación difícil de manejar
}

\author{
Pablo Zulet Fraile \\ Pedro Marcos Alberca \\ José Juan Gómez de Diego
}

Correspondencia

Pablo Zulet Fraile

pablo.zulet@salud.madrid.org

Instituto Cardiovascular, Hospital Clínico San Carlos, Instituto de Investigación Sanitaria del Hospital Clínico San Carlos (IdSSC), Madrid

Recibido: 10/09/2021.

Aceptado: 15/10/2021.

En línea: 15/12/2021.

Citar como: Zulet Fraile P, Marcos Alberca P, Gómez de Diego JJ. Trombo recurrente sobre dispositivo de cierre percutáneo de orejuela izquierda: una situación difícil de manejar. RETIC. 2021 (Diciembre); 4 (3): 47-49. doi: 10.37615/retic.v4n3a13

Cite this as: Zulet Fraile P, Marcos Alberca P, Gómez de Diego JJ. Recurrent thrombus on left appendage percutaneous closure device: a difficult situation to manage. RETIC. 2021(December); 4 (3): 47-49. doi: 10.37615/retic.v4n3a13

\section{Palabras clave}

$\triangleright$ Cierre percutáneo de orejuela izquierda.

$\triangleright$ Ecocardiograma transesofágico.

$\triangleright$ Trombosis de dispositivo de cierre de orejuela.

\begin{tabular}{l}
\hline Keywords \\
\hline$\triangleright$ Percutaneous closure of the \\
left appendage. \\
$\triangleright$ Transesophageal \\
echocardiogram. \\
$\triangleright$ Atrial Appendage Closure \\
Device Thrombosis. \\
\hline
\end{tabular}

\section{RESUMEN}

El cierre percutáneo de orejuela izquierda ofrece una alternativa a la anticoagulación en pacientes complejos con fibrilación auricular, por lo que uso se ha expandido en los últimos años. Sin embargo, la trombosis del dispositivo de cierre supone una complicación importante del procedimiento, que se ha estimado en un 3 a $4 \%$ de los casos según los registros publicados. Describimos el caso de una paciente de 85 años con historia de síndrome mielodisplásico que presentó trombosis recurrente sobre dispositivo un dispositivo de cierre de orejuela tipo Watchman ${ }^{\mathrm{TM}}$.

\section{Presentación del caso}

Mujer de 85 años con historia de hipertensión arterial, dislipemia, diabetes meIlitus tipo II y enfermedad renal crónica, en seguimiento por parte de Cardiología por fibrilación auricular paroxística ( $\mathrm{CHA}_{2} \mathrm{DS}_{2}-\mathrm{VASC} 5$, HAS-BLED 3), diagnosticada en 2018. Presentaba como antecedentes importantes un síndrome mielodisplásico bajo soporte hematológico con transfusiones quincenales por pancitopenia, y sangrado digestivo recurrente por angiodisplasias, motivo por el que se indicó el cierre percutáneo de orejuela izquierda (OI) para evitar la anticoagulación por alto riesgo hemorrágico.

Como parte del estudio previo al implante se realizó un ecocardiograma transesofágico (Vídeo 1), que mostró una orejuela izquierda (OI) de tipo "chicken wing" libre de trombos y se realizaron sus medidas para seleccionar el tamaño del dispositivo. El procedimiento se llevó a cabo bajo monitorización con ecocardiograma transesofágico, y se colocó un dispositivo de cierre de OI Watch- man $^{\top M}$ de forma exitosa (Vídeo 2). Tras ello, se inició tratamiento antiagregante con clopidogrel.

Se realizó un primer control con ecocardiograma transesofágico a los dos meses, donde se observó un trombo adherido al dispositivo de 0,8 ×0,7 cm (Vídeo 3). Tras discutir el caso con el servicio de Hematología, se decidió comenzar tratamiento anticoagulante con tinzaparina asociado al clopidogrel. Tras tres meses de anticoagulación, se comprobó la resolución del trombo (Vídeo 4), por lo que se retiró la anticoagulación con heparina de bajo peso molecular y se mantuvo antiagregación simple con clopidogrel. Dado el alto riesgo trombótico de la paciente, se realizó un nuevo control con ecocardiograma transesofágico que detectó de nuevo una masa sugestiva de trombo, pediculada y móvil, de 1,75 x 1,24 cm, adherida a la cara auricular del dispositivo Watchman ${ }^{\top M}$ (Vídeos 5-8). Tras este hallazgo, se retomó la estrategia de anticoagulación con heparina de bajo peso molecular (tinzaparina) y antiagregación con clopidogrel.

\section{Estudio por imagen}




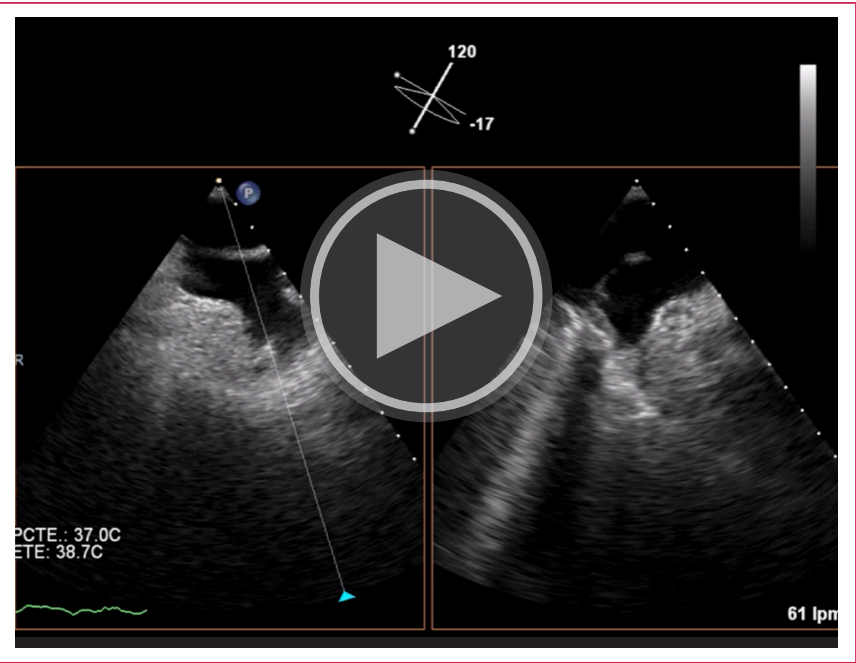

Vídeo 1. Ecocardiograma transesofágico (ETE): imagen de la orejuela izquierda en el estudio previo al implante del dispositivo de cierre.

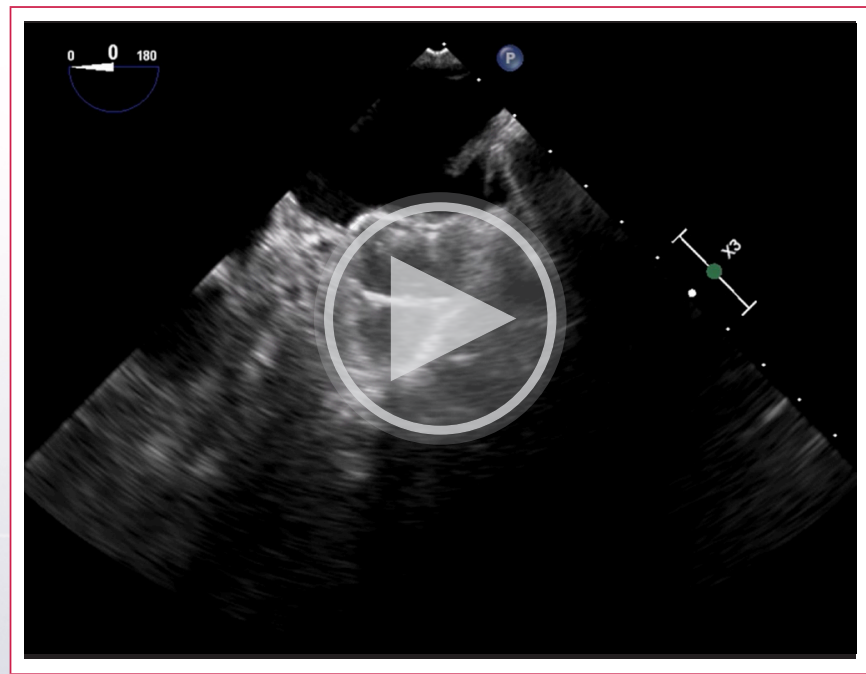

Vídeo 2. Ecocardiograma transesofágico (ETE): resultado del implante de dispositivo Watchman ${ }^{\text {TM }}$

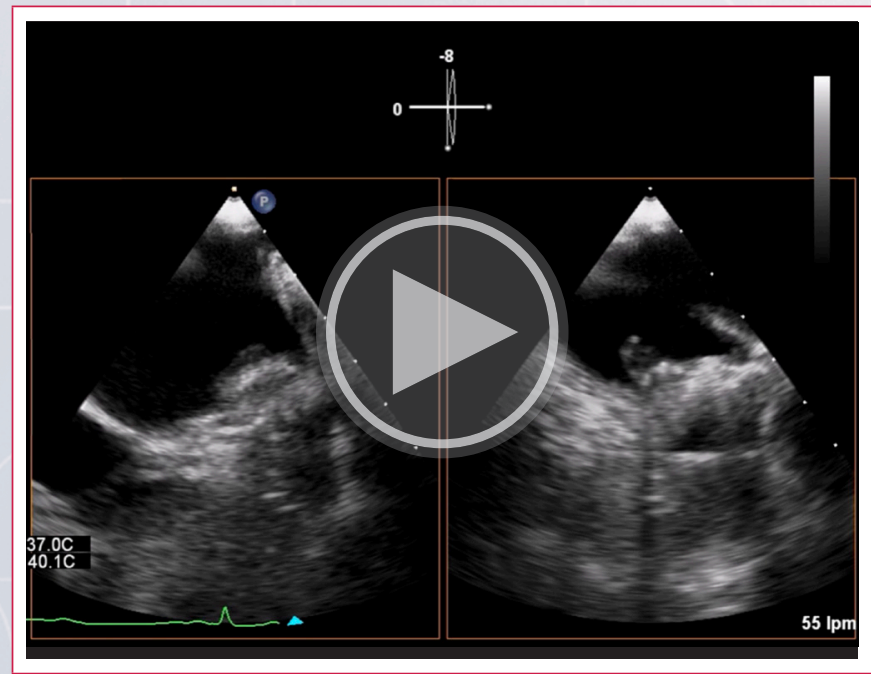

Vídeo 3. Ecocardiograma transesofágico (ETE): control a los tres meses del procedimiento, con imagen de trombo adherido a la cara auricular del dispositivo.

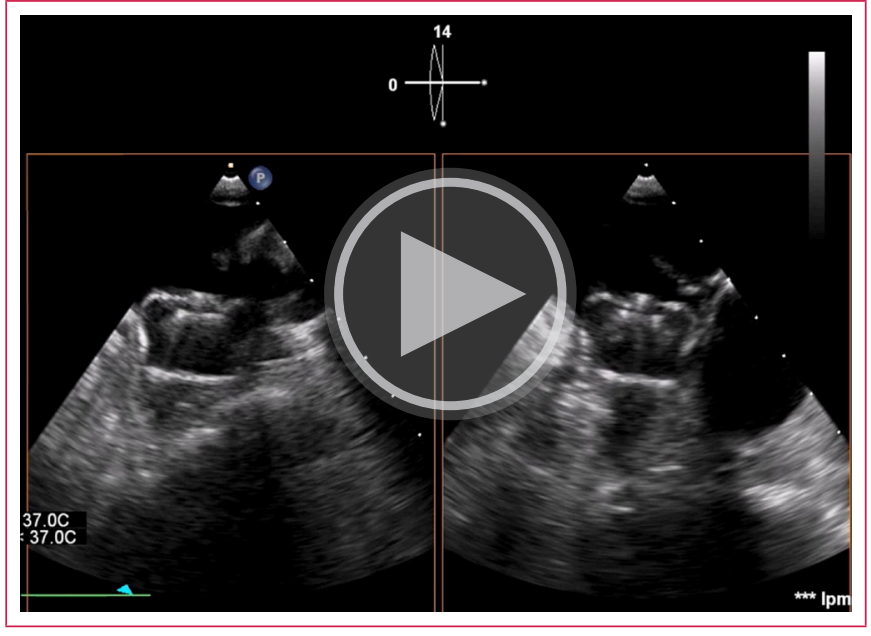

Vídeo 4. Ecocardiograma transesofágico (ETE): resolución del trombo tras tres meses de anticoagulación con heparina de bajo peso molecular y antiagregación simple.

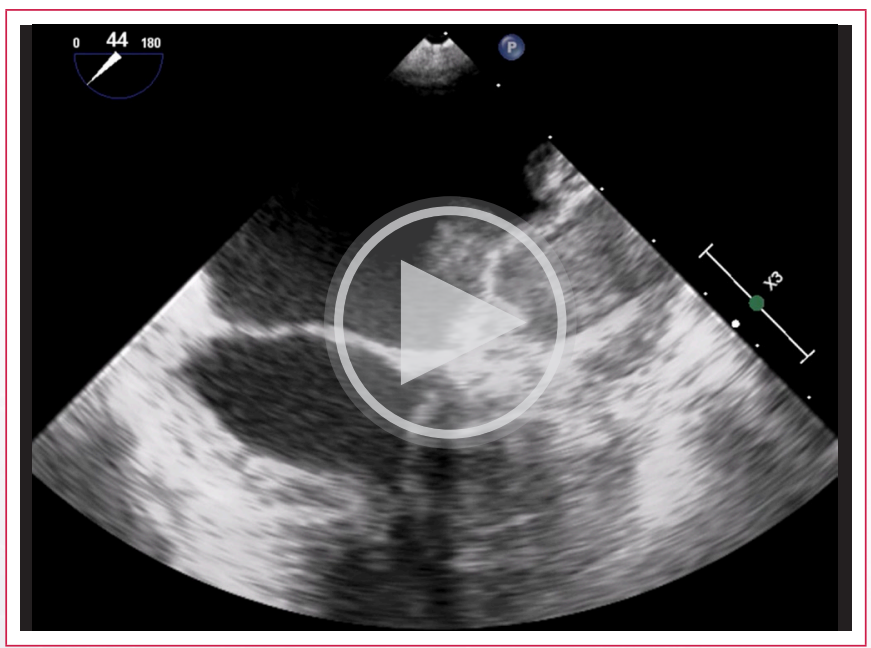

Vídeo 5. Ecocardiograma transesofágico (ETE): reaparición de una masa sugestiva de trombo pediculado y móvil adherido al dispositivo tras retirada de anticoagulación.

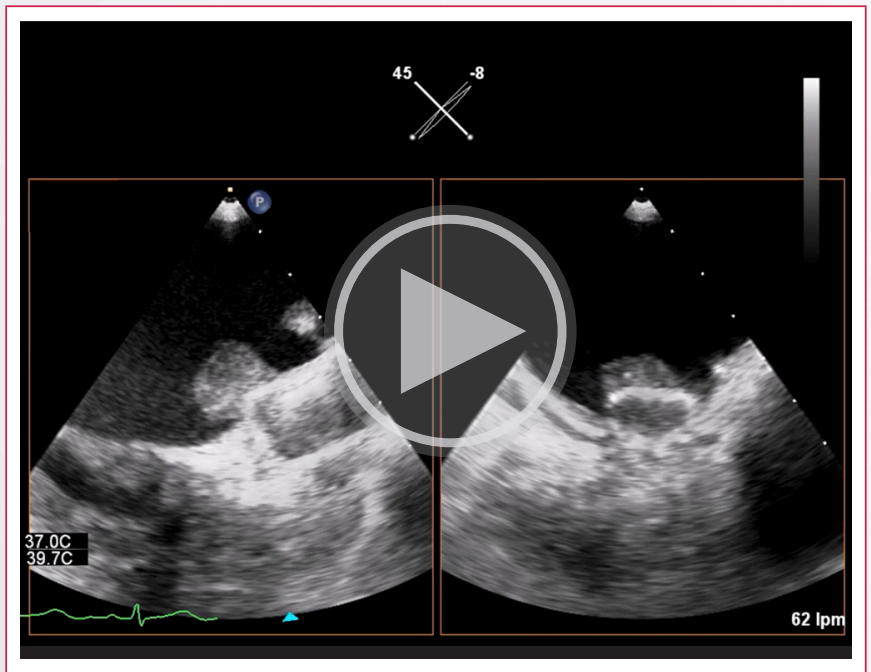

Vídeo 6. Ecocardiograma transesofágico (ETE): imagen con X-Plane que muestra con mayor detalle la masa sobre el dispositivo. 


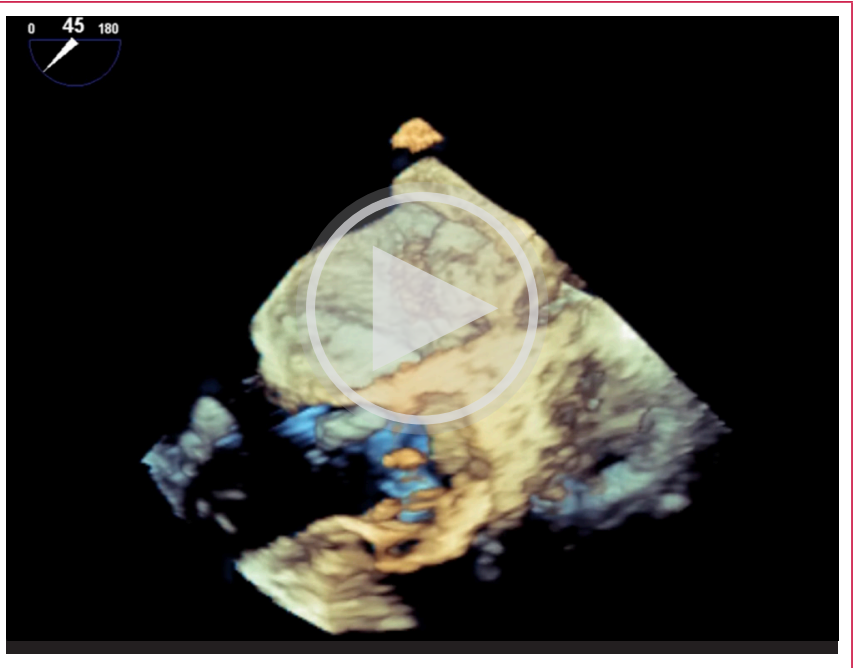

Vídeo 7. Ecocardiograma transesofágico (ETE): imagen con ecocardiografía $3 D$ en tiempo real. El 3D muestra la masa como una estructura redondeada y voluminosa de borde irregular en el cuadrante superior derecho de la imagen.

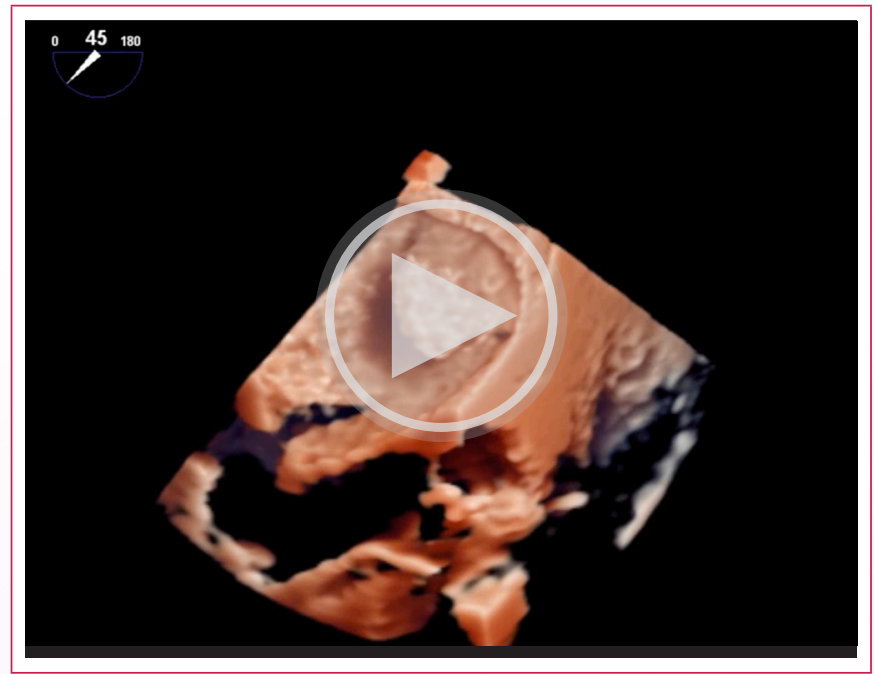

Vídeo 8. Ecocardiograma transesofágico (ETE): imagen con ecocardiografía 3D con el sistema TrueVue. Este procesamiento de imagen permite simular un "foco de luz" en la imagen y hacer que la masa sea todavía más evidente.

\section{Discusión}

El cierre percutáneo de la orejuela izquierda supone una alternativa en la prevención de riesgo tromboembólico en pacientes con fibrilación auricular con alto riesgo hemorrágico, debido a que reduce el riesgo de ictus hemorrágico y sangrado mayor en comparación con la estrategia de anticoagulación oral con warfarina'.

Sin embargo, se ha descrito que estos dispositivos de cierre se pueden trombosar. La trombosis asociada al dispositivo de cierre de OI resulta una complicación que supone un reto para el manejo del tratamiento antitrombótico, ya que generalmente estos pacientes parten de un elevado riesgo hemorrágico que fue la indicación del procedimiento.

En los registros multicéntricos su incidencia se ha estimado en un 3-4\% de los implantes, y en la mayoría de los casos se consigue su resolución con tratamiento anticoagulante oral. Sin embargo, si se forma un trombo en su superficie, el propio dispositivo de cierre supone una fuente embólica, y se ha descrito que la trombosis asociada a dispositivo de cierre de Ol aumenta el riesgo de eventos cardiovasculares, principalmente a expensas de aumento de la incidencia de ictus isquémico ${ }^{2-3}$.

Se ha intentado determinar cuáles son los factores de riesgo para desarrollar esta complicación, algunos de ellos asociados al paciente y otros al procedimiento. Los trastornos de hipercoagulabilidad, la insuficiencia renal crónica, la fibrilación auricular no paroxística, el derrame pericárdico tras el procedimiento, y la profundidad del implante a más de $10 \mathrm{~mm}$ del limbo de la vena pulmonar suponen los factores de riesgo más importantes ${ }^{3-5}$

El ecocardiograma transesofágico resulta la herramienta principal tanto para guiar el procedimiento y disminuir los factores de riesgo relacionados con el mismo, como para el diagnóstico y seguimiento de la trombosis asociada al dispositivo. La tomografía computarizada (TC) cardiaca resulta una alternativa con alta rentabilidad para el diagnóstico de esta complicación6.

\section{Conclusión}

El cierre percutáneo de orejuela izquierda supone una alternativa terapéutica en el manejo del riesgo tromboembólico asociado a la fibrilación auricular que no está exenta de complicaciones asociadas. La trombosis recurrente sobre el dispositivo se asocia a estados de hipercoagulabilidad, y puede ser un reto terapéutico especialmente en casos de alto riesgo hemorrágico. El ecocardiograma transesofágico es la prueba de elección tanto para guiar el procedimiento de cierre percutáneo de la orejuela izquierda como para el diagnóstico y seguimiento de la trombosis asociada al dispositivo.

\section{Ideas para recordar}

- La trombosis asociada a dispositivo de cierre de orejuela izquierda se ha descrito en un 3-4\% de los casos.

- Los trastornos de hipercoagulabilidad, la insuficiencia renal crónica, la fibrilación auricular no paroxística, el derrame pericárdico tras el procedimiento, y la profundidad del implante a más de $10 \mathrm{~mm}$ del limbo de la vena pulmonar suponen los factores de riesgo más importantes.

- La trombosis asociada a dispositivo de cierre de orejuela izquierda aumenta el riesgo de eventos cardiovasculares, principalmente a expensas de ictus isquémico.

\section{Bibliografía}

1. Reddy VY, Doshi SK, Kar S, et al., PREVAlL and PROTECT AF Investigators. 5-Year outcomes after left atrial appendage closure. J Am Coll Cardiol 2017;70:2964-75.

2. Alkhouli M, Busu T, Shah K, Osman M, Alqahtani F, Raybuck B. Incidence and clinical impact of device-related thrombus following percutaneous left atrial appendage occlusion: a meta-analysis. J Am Coll Cardiol EP 2018;4: 1629-37.

3. Trevor Simard,MD, Richard G. Jung, BSC, et al. Predictors of Device-Related Thrombus Following Percutaneous Left Atrial Appendage Occlusion. J Am Coll Cardiol. 2021 Jul 27;78(4):297-313.

4. Dukkipati SR, Kar S, Holmes DR, et al. Device related thrombus after left atrial appendage closure. Circulation 2018;138:874-85.

5. Kaneko H, Neuss M, Weissenborn J, Butter C. Predictors of thrombus formation after percutaneous left atrial appendage closure using the WATCHMAN device. Heart Vessels 2017;32: 1137-43.

6. Korsholm K, Jensen JM, Nørgaard BL, Nielsen-Kudsk JE. Detection of devicerelated thrombosis following left atrial appendage occlusion. Circ Cardiovasc Interv 2019;12:e008112. 